\title{
VERS UNE CULTURE DE LA DIVERSITE'
}

\author{
M’Badi Miehakanda
}

Université des Antilles et de la Guyane / Université de Nancy 2

\section{Résumé}

Cet article porte sur les pratiques langagières en cours dans les crèches et les écoles maternelles martiniquaises. Il s'intéresse au repère des modalités relatives aux interactions quotidiennes dans ces cadres et plus précisément aux distorsions langagières qui peuvent intervenir lors des échanges entre adultes et enfants. La prise en compte des diversités émanant des distorsions langagières pourrait constituer un préalable au développement d'une dynamique de compréhension réciproque nécessaire à la maîtrise des conduites langagières et à l'apprentissage chez les tout petits en Martinique.

Ce texte propose de nouvelles modalités dans le cadre d'un contrat didactique respectueux des différences.

Mots clé : interactions -distorsions langagières- représentations - diversité culturelle - intercompréhension

\section{TOWARD A CULTURE OF DIVERSITY}

\begin{abstract}
This paper deals with language practices in nurseries and pre-elementary schools of Martinique. It is focused on the account of modalities concerning everyday interactions and more precisely language distortions that may occur during interactions between adults and children in those structures. The consideration of cultural diversity generated by language distortions is suggested here to be a prerequisite for the development of a reciprocal understanding leading to adequate mastery of linguistic behavior and learning among young children.

This approach proposes new patterns within a didactic agreement considering adult and child differences.
\end{abstract}

Key words: interactions - language distortion- representation - cultural diversity - reciprocal comprehension

Université des Antilles et de la Guyane / Université de Nancy 2

97200 Fort de France - MARTINIQUE - Tél. : 0696944974 - Courriel : miehakandambadi@yahoo.fr 


\section{INTRODUCTION}

Pour appréhender l'entrée de l'individu dans la culture, Bruner (1996) propose l'idée d'une éducation susceptible d'adapter une culture aux besoins de ses membres et d'adapter ses membres et leurs manières d'apprendre aux besoins de la culture. Selon lui, nos actions sont guidées par des valeurs, des normes qui, loin d'être "naturelles", sont des constructions culturelles et symboliques (Bruner, 1999). Il conçoit le développement humain comme un processus de collaboration entre enfant et adulte, l'adulte étant envisagé comme médiateur de la culture.

Des distorsions langagières interviennent cependant dans les interactions adultes-enfants en raison d'une asymétrie entre leurs niveaux d'expérience respectifs dans la langue parlée, mettant en évidence les disparités entre les intentions de ces interlocuteurs (Miehakanda, 2010). Ces disparités sont en rapport avec des diversités culturelles, sociales et individuelles intervenant dans la transmission des savoir-faire langagiers et l'intercompréhension qu'elle implique.

La présente réflexion propose de mettre en perspective les conditions d'application d'une telle approche en Martinique. Elle sera introduite par une présentation du contexte culturel et linguistique dans lequel évoluent les locuteurs en Martinique. Les valeurs socioculturelles véhiculées par les adultes qui encadrent les enfants seront prises en compte dans une approche des positionnements communément adoptés dans les établissements vis-à-vis de la norme sociolinguistique. Cette norme s'inscrit dans une politique linguistique qu'il convient d'expliciter pour appréhender les orientations sociales qu'elle induit. La diversité culturelle des lieux d'implantation de la langue française est un élément essentiel de cette politique linguistique et l'élaboration d'un nouveau contrat didactique s'y impose dans la perspective d'une régulation des interactions adultes-enfants en milieu francophone / créolophone.

\section{APPROCHE DU CONTEXTE CULTUREL ET LINGUISTIQUE MARTINIQUAIS}

Les Martiniquais parlent généralement le français et le créole, le premier idiome étant la forme linguistique acrolectale (langue haute) adoptée comme la norme linguistique, le second correspondant à la forme basilectale (langue basse) employée le plus souvent dans les échanges familiers et de proximité. Bernabé (1984) propose à ce sujet un modèle qui se situe dans un continuum / discontinuum où chaque langue (le créole et le français) est dotée d'un acrolecte et d'un basilecte. 
Les locuteurs de cette région sont confrontés aujourd'hui à la fois à l'influence de la langue française établie comme la norme et à une présence sous-jacente à l'école de la langue créole pratiquée dans leur environnement langagier.

Cette situation sociolinguistique donne lieu à des productions qui se situent parfois à mi chemin entre créole et français : on parle alors d'une créolisation du français ou d'une francisation du créole, le 'code mixing' et le 'code switching' étant par ailleurs devenu des pratiques langagières courantes. Prudent (1993) attire l'attention sur la notion d'interlecte qui désigne la zone de superposition du créole et du français où les locuteurs martiniquais se jouent de la norme grammaticale en faisant usage de lectes ${ }^{2}$ non hiérarchisés dans leurs pratiques langagières. L'analyse du contexte écolinguistique martiniquais est de ce fait rendue complexe par la difficulté qu'on éprouve à distinguer des locuteurs francophones et des locuteurs créolophones.

Il semble opportun de s'interroger sur les modes de transmission et d'appropriation des savoir-dire pour tenter de comprendre comment les enfants apprennent à parler dans de telles conditions, et d'identifier les pratiques langagières dans les situations de communication adultes enfants où ces derniers font leurs premières expériences sociales du langage oral.

Un des objectifs de cette réflexion est de découvrir l'opportunité d'une éventuelle prise en compte de la langue créole au contact de la langue française lors de la transmission des savoir-dire dans l'environnement sociolinguistique martiniquais marqué par l'emploi sélectif de deux idiomes vecteurs de valeurs socioculturelles différentes.

Pour appréhender les caractéristiques de ce contexte, une pédagogie fondée sur les capacités adaptatives des apprenants et leurs aptitudes à co-construire leur espace linguistique à l'intérieur de rituels langagiers clairement identifiés peut constituer un terrain favorable à une répartition rationnelle des fonctions du français et du créole, comme le démontrent des expériences hélas encore trop marginales dans le milieu scolaire (actions volontaires d'enseignants militants).

Les tout petits en Martinique, comme leurs pairs à travers le monde, contribueront-ils un jour, à travers les interprétations spontanées des pratiques langagières qui leurs sont apportées par les adultes, à la mutation vers une plus grande homéostasie de l'espace écolinguistique dans lequel ils évoluent?

\footnotetext{
${ }^{2}$ Un lecte est un faisceau de variantes relatives à l'emploi d'une langue dans une communauté.
} 


\section{Orientation linguistique et représentations sociales.}

Les directrices des crèches visitées sont unanimes à reconnaître que les exigences relatives à l'emploi exclusif de la langue française n'émanent d'aucune législation et ne font pas non plus partie du cahier des charges de leurs organismes de tutelle. Cette orientation linguistique pourtant rigoureusement appliquée dans l'ensemble des établissements est, selon leurs propres dires, prioritairement influencée par leurs représentations des langues de leur environnement (ex : créole=patois) et par l'éducation qu'elles ont reçue. Elles indiquent également l'implication des attentes des familles qui leur confient leurs enfants, en particulier celles des parents d'origine martiniquaise pour qui la maîtrise du français représenterait un atout majeur dans l'éducation des tout petits. Une directrice d'école maternelle située en zone résidentielle témoigne des résistances des enseignants de son établissement à utiliser les supports en langue créole mis à leur disposition dans le cadre d'expressions poétiques. Une de ces personnes corrobore son positionnement réfractaire en affirmant qu'elle n'accepterait d'utiliser ces supports que si elle y était contrainte, ce qui donne à réfléchir sur les rapports qu'elle entretient avec cet idiome qui est pourtant l'un des siens. Cela nous donne en tout cas un aperçu des intentions éducatives et des représentations linguistiques d'adultes chargés d'instruire les enfants en Martinique.

Le positionnement des Martiniquais à l'égard de leurs langues semble pour le moins ambivalent lorsqu'il s'agit d'éduquer et d'instruire les enfants. Il leur est difficile d'employer le créole comme langue de l'éducation et de l'instruction alors qu'ils se reconnaissent locuteurs de deux idiomes, le français et le créole qu'ils parlent couramment. Il convient donc de s'arrêter sur les représentations des langues par leurs locuteurs en Martinique, car comme dans toute autre société, les représentations sociales interviennent activement dans la transmission et l'appropriation des pratiques langagières.

L'ensemble de la communauté scientifique identifie la représentation sociale comme une forme de connaissance, socialement élaborée et partagée, ayant une visée pratique et concourant à la construction d'une réalité commune à un ensemble social (Jodelet, 1989, Les représentations sociales, page 36). Les représentations que les locuteurs se font des langues, de leurs normes, de leurs caractéristiques, ou de leurs statuts influencent les procédures et les stratégies qu'ils développent et mettent en œuvre pour les apprendre et les utiliser, selon Dabène (1997). 
Candelier et Hermann-Brennecke (1993) montrent dans leur approche des motivations de choix et d'abandon des langues étrangères par les étudiants français et allemands que le contact scolaire avec la langue influence de manière positive les représentations attachées à cette langue. En Martinique le contact scolaire avec le créole est quotidien en dépit de l'omniprésence du français qui est la langue de l'enseignement : les interdits et les censures dont fait l'objet le créole n'ont pas raison de sa présence, même allusive, dans les discours des adultes-éducateurs.

Une prise en compte du créole à l'école permettrait sans doute à cette langue de bénéficier du préjugé positif que mentionnent Candelier et Hermann-Brennecke. Ceux-ci ont constaté en effet que l'évolution des représentations peut être altérée par le degré de pertinence que les élèves allouent à la langue de l'enseignement, à travers leurs rapports imaginés ou effectifs à leur contexte sociolinguistique. Il importe alors de poser le problème sous l'angle de la norme linguistique instituée par l'école qui a une incidence sur les représentations sociales, notamment en termes de conduites langagières, et qui s'inscrit dans le cadre d'une politique linguistique française où les langues régionales comme le créole ne sont pas encore du domaine des usages officiels en dépit des récentes réformes ${ }^{3}$.

\section{Incidences de la politique linguistique actuelle.}

La France est l'un des pays les plus réservés face à la ratification de la charte européenne des langues régionales ou minoritaires qui confère à celles-ci une légitimité relative à leur présence sur leurs territoires d'implantation.

Dans son article 8, cette charte stipule notamment que les parties signataires s'engagent à prévoir qu'une partie substantielle de l'éducation préscolaire soit assurée dans les langues régionales ou minoritaires concernées ; à prévoir un enseignement primaire assuré dans ces langues.

En France, le conseil constitutionnel s'est toujours s'opposé à ce qu'il considère comme une menace pour la langue de la République, les langues régionales ou minoritaires pouvant concurrencer voir dévaluer la langue française si on les introduisait au même titre dans la constitution. La langue de la République est le français, est-il stipulé dans l'article 2 de la constitution, ce sur quoi se fondent les réserves des opposants à la ratification de la charte.

\footnotetext{
${ }^{3}$ La nouvelle constitution reconnaît les langues "régionales" comme appartenant au "patrimoine de la France". Depuis 2008, l'article 75-1 de la Constitution française de 1958 reconnaît les langues régionales comme appartenant au patrimoine de la France. Aucune n'a cependant statut de langue officielle, qui est celui du seul français.
} 
Les résistances de ceux-ci face à la récente modification de cet article sont liées aux craintes d'une remise en question de la souveraineté nationale d'autres instruments y figurant, à savoir le drapeau, l'hymne, la devise et le français.

Les conclusions de la réforme constitutionnelle qui s'est déroulée en mai 2008 sous l'insistance du Président de la République permettent en effet d'introduire les langues régionales ou minoritaires dans le patrimoine linguistique français. Mais cette réforme votée par l'Assemblée Nationale a fait l'objet d'un amendement du Sénat qui a refusé d'inscrire la reconnaissance des langues régionales dans la constitution.

Le 22 mai 2008, l'Académie Française a de plus exprimé son opposition à la reconnaissance des langues régionales qu'elle considère comme une atteinte à l'identité nationale. Elle a purement et simplement demandé le retrait de l'article les concernant.

La politique linguistique française tend donc à se figer sur d'anciens modèles concernant sa langue nationale et le patrimoine qu'elle lui associe, à l'exclusion des autres langues parlées sur ses différents lieux d'implantation. Dans ce contexte, la langue créole n'est que partiellement introduite dans les écoles martiniquaises, notamment à travers la formation de certains professeurs au Certificat d'Aptitudes au Professorat de l'Enseignement du Second degré (CAPES) créole et d'autres enseignants aux Langues et Cultures Régionales (LCR), sous l'impulsion entre autres du Groupe d'Etude et de Recherche en Espace Créolophone et Francophone (GEREC-F), devenu Centre de Recherche Interdisciplinaire en Littérature, Langues, Art et Sciences Humaines (CRILLASH).

La langue française demeure la seule langue de l'enseignement, le créole ayant jusqu'ici une implication optionnelle dans les apprentissages scolaires inscrits dans des programmes où il s'agit plus d'enseigner la langue régionale que d'enseigner en langue régionale.

Cela revient à dire qu'une politique linguistique véritablement orientée vers une prise en compte des diversités culturelles des régions concernées par les rapports langue française/langue régionale doit mettre en place les conditions optimales d'expression de cette diversité en répartissant notamment les champs d'intervention de chaque langue à la fois en matière de communication (aspect social) et de pédagogie (aspects disciplinaires).

En pratique, tous les enseignants et tous les professionnels de la petite enfance devraient se former à une maîtrise équivalente du français et de leur langue régionale respective dans leurs dimensions fonctionnelles (expression orale et expression écrite) mais aussi en tant qu'outils didactiques (organe véhiculaire des connaissances). 
Il s'agirait, entre autres, d'être en mesure de mettre en perspective un idiome par rapport à un autre (le créole par rapport au français et réciproquement) lors de l'exploration des concepts et savoir-faire à transmettre dans tous les domaines propres aux apprentissages préscolaires et scolaires.

De telles dispositions ont un double intérêt: celui d'organiser le processus d'intercompréhension et les rencontres intersubjectives qu'il implique autour d'une prise en compte des disparités socioculturelles liées à la présence des deux idiomes dans les environnements langagiers des jeunes locuteurs ; celui d'orienter les pratiques langagières des écoliers martiniquais vers un usage bilingue du français et du créole qui serait en rupture avec l'monolinguisme actuellement pratiqué dans l'espace éducatif français.

L'adoption le 10 avril 2009, dans le cadre de la Loi pour le Développement Economique de l'Outre Mer (LODEOM), d'un amendement introduisant les langues créoles dans le patrimoine national français permet d'espérer que de nouvelles dispositions administratives sauront accueillir de nouvelles modalités pédagogiques adaptées à la mise en œuvre d'une pratique harmonieuse du créole et du français, notamment parmi les tout petits fréquentant les structures préscolaires et scolaires en Martinique.

\section{Diversités culturelles et diversités langagières.}

Dans la plupart des régions extra-hexagonales concernées par une présence du français en tant que langue officielle, apprendre à parler en collectivité et à l'école tout en préservant son identité culturelle requiert des conditions didactiques et sociales qui doivent être construites au contact d'un langage commun impliquant les composantes culturelles et sociales de l'environnement de chaque locuteur en présence.

L'intérêt d'une telle approche des pratiques langagières, à la Martinique par exemple, est lié à une diversité des expressions culturelles d'une société, chaque langue, le créole comme le français, étant le vecteur d'une culture et d'un mode de pensée distincts. Le créole fait référence à une culture renvoyant à une tradition orale issue en grande partie d'un vécu dans les plantations coloniales tandis que le français fait référence à une culture de l'Europe occidentale, plus ancienne, où les concepts sont depuis longtemps retranscrits en langage écrit.

Cela se traduit aujourd'hui par des pratiques langagières reproduisant les diversités culturelles des locuteurs martiniquais qui, suivant leur éducation et les milieux sociaux dans lesquels ils évoluent, s'approprient le créole et le français à travers des usages qui leur sont propres. 
De ce fait on ne rencontre guère de Martiniquais s'exprimant exclusivement en français ou en créole. On peut ainsi constater que ces deux idiomes sont inscrits, compte tenu (ou en dépit) de conditions de diglossie encore prégnantes, dans le patrimoine linguistique des locuteurs martiniquais.

Selon Charaudeau (2001), la langue est, certes, nécessaire à la constitution d'une identité collective mais il convient de dissocier langue et culture, et d'associer plutôt les usages langagiers à la culture. Cette distinction est repérable dans les rituels langagiers et dans le contrat didactique entre enseignants et élèves en Martinique où les premiers font référence au créole de manière plus ou moins indirecte, mais aussi dans les disparités entre les usages langagiers des enfants et ceux des adultes chargés de leur éducation. Il est, en effet, plusieurs manières de parler une même langue si on considère d'une part le niveau de maturation du locuteur, d'autre part le(s) milieu(x) socioculturel(s) dans lequel (lesquels) il évolue habituellement.

Ainsi, lorsqu'il s'agit de locuteurs amenés à communiquer dans une langue qui n'est pas leur langue d'origine, on se trouve en présence d'une rencontre de modes de pensée différents car chaque locuteur est non seulement porteur d'une identité construite au contact de l'idiome qu'il emploie mais il est aussi dépositaire des usages se rattachant à la culture de son entourage langagier.

Les intentions respectives des personnes impliquées dans l'interaction sont fonction non seulement des motivations en lien direct avec les situations de communication et leurs conditions d'intercompréhension mais aussi des modalités d'utilisation de l'idiome qu'ils emploient habituellement dans leurs milieux sociaux et familiaux respectifs.

En ce qui concerne l'emploi du créole et du français en Martinique, nous pouvons évoquer ici la situation d'ancrage qui fait que chacun de ces idiomes tient une place privilégiée par rapport à l'autre suivant les situations sociales vécues par les locuteurs.

C'est à la faveur de cet ancrage que les locuteurs martiniquais s'approprient sur un mode identitaire l'une et l'autre de ces langues qui constituent les marquages culturels de leur environnement sociolinguistique. 


\section{Une culture de la diversité (implications didactiques).}

Ce n'est donc plus à l'exclusion l'une de l'autre que la langue française et la langue créole doivent être apprises en Martinique mais, bien au contraire, l'une au contact de l'autre, dans une perspective didactique favorisant la prise en compte mutuelle des disparités culturelles liées à l'emploi respectif de chaque idiome. Prudent propose de fonder une pédagogie du français et du créole en prenant en compte la zone interlectale 4 qui est au cœur de la langue maternelle du Martiniquais (Prudent, 2010, page 7) pour prendre en compte l'élève martiniquais et son environnement sociolinguistique. Ceci constitue, dans le domaine de la didactique des langues, une grande avancée qui permet d'appréhender la variété des lectes employés dans chaque idiome par ces jeunes locuteurs en situation d'apprentissage.

Les modalités relationnelles en matière d'acquisition langagière sont toutefois parcourues par des rituels qui peuvent, à la faveur d'une médiation stratégique de l'enseignant, mettre en question l'implication des deux idiomes dans les différentes situations d'apprentissage. Dans les crèches et les écoles maternelles, cela devrait se traduire par une posture d'accueil et de transmission multiculturels de l'adulte vis-à-vis des enfants qu'il encadre. La posture d'accueil correspond à une attitude d'écoute et d'observation des conduites langagières spontanées des tout petits encadrés par les adultes. Le fruit de cette démarche est à mettre au bénéfice d'un repérage et d'un recueil des disparités d'ordre social et culturel.

La posture de transmission correspond à une prise en compte des disparités repérées et à une exploitation de celles-ci lors de la construction collective des savoir-faire. Les productions créoles et françaises sont réinvesties dans l'ensemble des interactions. Le contrat didactique encadrant-encadré, enseignant-enseigné se concentre sur les modalités mises en œuvre en situation d'apprentissage. Dans ce contexte, les élèves sont invités à expliciter et argumenter leurs propres productions et celles de leurs pairs, la consigne principale étant de s'exprimer librement en français ou en créole.

Ce dispositif pédagogique requiert, il convient de le rappeler, une formation commune des adultes-référents et des enseignants en langue régionale (orale et écrite) mais aussi en didactique des langues, de manière à acquérir des compétences en matière d'enseignement bilingue et de repère des usages langagiers. Il est également essentiel que ces acteurs de la petite enfance soient compétents dans le domaine de l'observation des conduites langagières.

\footnotetext{
${ }^{4}$ Entre le basilecte et l'acrolecte.
} 
A terme, c'est une sensibilisation et une formation des tout petits aux disparités et diversités culturelles de leur environnement langagier qui sont posées comme des principes éducatifs, notamment dans les situations d'apprentissage où les savoir-faire se construisent aussi bien au contact des adultes que des pairs, toutes compétences linguistiques confondues.

Lorsque, par exemple, une des directrices de crèche fait la présentation des activités de son établissement, elle précise qu'il est fréquenté par des enfants dont les origines culturelles s'avèrent particulièrement diverses en raison, notamment, de sa zone d'implantation.

Située dans un quartier où résident des familles martiniquaises, françaises, italiennes, allemandes, cambodgiennes et togolaises, cette crèche de la périphérie de Fort de France accueille des enfants qui, au contact de disparités culturelles, doivent évoluer ensemble de la section des tout petits à la section des grands. Cette directrice, pour prendre en compte les différences interculturelles, organise régulièrement un tour $d u$ monde des cultures avec, à chaque voyage, la contribution active d'une famille culturellement impliquée. Lors de ces rencontres, les enfants découvrent les cultures les uns des autres au travers d'activités ludiques (ex : jeux typiques des pays visités) et au moyen d'animations culinaires, musicales, dansantes et linguistiques. Les adultes référents interviennent en médiateurs, en fournissant aux enfants de nouveaux outils langagiers pour communiquer avec leurs pairs en se référant à des registres culturellement diversifiés (ex : chansonnettes de contrées différentes) dans le cadre de rapports fondés sur une construction interculturelle des savoir-faire très bien accueillie par les enfants. Cet exemple montre l'intérêt du respect des différences interindividuelles pour le développement d'un processus d'intercompréhension favorable à l'épanouissement langagier des jeunes locuteurs. En effet, les tout petits mis en situation de découverte avec leurs pairs par un adulte référent soucieux de ce respect des différences parviennent à construire les savoir-faire qui leur sont transmis en mettant en œuvre leur propre expérience langagière (intra subjective) tout en s'enrichissant de celles de leurs petits camarades (expérience intersubjective).

C'est en valorisant et en stimulant l'expression et la réception des différences culturelles parmi les jeunes locuteurs dont il a la charge éducative que l'adulte devrait obtenir les conduites langagières les plus adaptées et les plus performantes. Les diversités culturelles sont à mettre au bénéfice du processus d'intercompréhension par un adulte-référent qui doit créer les conditions optimales d'échanges parmi les jeunes locuteurs dont il a la charge. Il doit pour cela tenir compte à la fois des capacités et potentialités des enfants et de l'environnement sociolinguistique où ils évoluent. 


\section{BIBLIOGRAPHIE}

Bernabé, J., Chamoiseau, P., Confiant, R., 1989, Éloge de la créolité, Paris, Gallimard.

Bernabé, J. (1983): Fondal-natal: Grammaire basilectale approchée des créoles guadeloupéen et martiniquais. Paris, L'Harmattan.

Bernicot, J. (1996), Les actes de langage chez l'enfant (Paris : PUF)

Bruner, J. (1996), L'éducation, entrée dans la culture. Les problèmes de l'école à la lumière de la psychologie culturelle. Paris : Retz.

Bruner, J. (1999). Rencontre avec Jérôme Bruner, Propos recueillis par Dortier J. F. Pour une psychologie culturelle, Sciences humaines XXX, mensuel n ${ }^{\circ} 99$.

Candelier, M., Hermann-Brennecke, G. (1993). Entre le choix et l'abandon : les langues étrangères à l'école, vues d'Allemagne et de France, dans Représentations sociales des langues et enseignements, Guide pour l'élaboration des politiques linguistiques, chap. 2 p. 11 Charaudeau, P. (2001), Langue, discours et identité culturelle, Revue de didactologie des langues - cultures, 2001/3-4 - Nº123 ISSN 0071- 190X | ISBN | pages 341 à 348.

Dabène, L. (1997), L’image des langues et leur apprentissage. In M. Matthey (Ed.), Les langues et leurs images, Neuchâtel : IRDP, 1997, op cité 19-23.

Gumperz, J. (1989). Sociolinguistique interactionnelle : une approche interprétative. Paris: L'Harmattan.

Jodelet, D. (1984). Les représentations sociales. Paris : PUF

Miehakanda, M. (2010), Transmission et appropriation des langues en Martinique (Université des Antilles et de la Guyane, Martinique. Thèse de doctorat.

Moscovici, S. (1976). La psychanalyse, son image et son public. Paris : P.U.F. (2 ed.).

Prudent, L.F. (1993). Pratiques martiniquaises : genèse et fonctionnement d'un système créole. Université de Haute-Normandie : Thèse de Doctorat d'Etat.

Prudent, L.F. (2010). Adaptation de la didactique du français aux situations de créolophonie.. Guide du maître, Organisation Internationale de la Francophonie, LeWebPédagogique, La Martinique. 